\title{
Recent News in Medical Nutrition Therapy
}

\author{
Istvan G Télessy* \\ University of Pécs, Faculty of Pharmacy, Department of Pharmaceutics, Hungary \\ *Corresponding author: Istvan G Télessy, PharmD, Associate professor of the Department of Pharmaceutics, University of Pécs, 2100 Gödöllő, Fácán sor 25, Hungary
}

Received: November 21, 2021; Accepted: November 29, 2021; Published: December 20, 2021

\begin{abstract}
Medical nutrition is essential part of the medical therapy. Undernourished patients predict worse outcome in various disease states and higher costs for the healthcare system. Research activity and presentation of best practices ensure the continuous development of this discipline. Here, some selected news are introduced from the last 4-6 years. The GLIM criteria serve as internationally accepted uniform tool for assessment of patients nutritional status instead of the several assessment tools applied before. In the field of parenteral nutrition huge development was the introduction of the GLP-2 agonist teduglutide that help short bowel patients to the gut adaptation. An other discovery was the indicator function of citrulline in the same patient group. Some new recognitions in the field of macronutrients amino acids and lipid emulsions are also discussen. Finally three preactical innovations of enteral nutrition are negotiated: the recommended use of supplemental parenteral nutrition for patients where planned macronutrient supply can not be reached via enteral nutrition, the bioavailability of amino acids administered orally or enterally which is wors than previously conceived and the use of citrulline in the oral and enteral nutrition is recommended due to its multiple benefits and good bioavailability.
\end{abstract}

Keywords: Medical nutrition, Parenteral nutrition, Enteral nutrition, GLIM criteria, Teduglutide, Amino acid, Fat emulsion, Citrulline

\section{Introduction}

Nutrition therapy is a dinamically developing specialty. One important part of it, the medical nutrition therapy obviously must be part of the therapeutic armamentarium because undernutrition definitively worsens patients outcome, elongate recovery time and increase treatment costs. Some of the recent findins influenced the strategy and/or daily practice of this discipline. Also, appearance of precision medicine influenced the doctors attitude in this aspect; therapeutic consideration became more accurate and carefull. Here we gethered highlights of the nutrition therapy of the last ca. 5 years and present in a condensed form focusing to the parenteral and enteral nutrition.

\section{Assessment of Nutritional Status of Patients}

During the past decades many assessment tools have been developed. Most used ones are the NSN2000, the NRS-2002, the SGA, the MUST, the MNA and mini-MNA, etc. Most of them are specifically good in certain patient population and bear weaknesses in other fields. Internationally, in 2016 started a discussion among leaders of various potent nutrition-oriented scientific societies about development of a new tool enabling global use with global consensus. By 2019 GLIM criteria was elaborated [1].

GLIM criteria (Global Leadership Initiative on Malnutrition) is a two-step evaluation of patients' parameters having risk of undernutrition. First step is an assessment of patients' parameters with one of the previously used screening methods. Those who are at risk for undernutition according to these assesments, should be subjects of the second assesment. In this $2^{\text {nd }}$ phase three phenotypic and two ethiologic criteria are assessed. Just one criterion should be present from both group of criteria to declare diagnosis of undernutrition. Phenotypic criteria are either accidental and tendencious loss of weight or BMI under 18.5 or reduction of muscle mass. Ethiologic criteria are reduced food intake or inflammation or presence of devastating disease.

The usefulness of this tool has been tested in various patient and disease categories during the last 2 years and are running as well [2-4]. To date, the correspondence with empirical results and declaration of undernutrition according to the GLIM Criteria has been confirmed [5].

\section{Parenteral Nutrition}

Parenteral Nutrition (PN) is one of the most risky way of antificial nutrition even if this risk is less than that of several intravenous medications. Parenteral nutrition may provide a more risky nutritional form than Enteral Nutrition (EN), terefore EN is the preferred route of administration however, in certain situation this is unavoidable and certainly more efficient that EN. The very first example of this $\mathrm{PN}$-dependent condition is the Small Bowel Syndrome (SBS). In this theme most recent development was the introduction of teduglutide.

\section{Introduction of Teduglutide into the Daily Routine}

Teduglutide is a synthetic analogue of glucagon like peptide-2 (GLP-2). Very similar to GLP-1 agonists, which are successfully used for ca. a decade in the diabetes therapy, as this incretine hormone is produced in the small intestine. The GLP-2 hormone is also produced in the enteroendocrine L-cells of the lower Gastrointestinal Tract (GIT), closer in the ileum and colon and its receptors are located in the same gut-segment. GLP-1 and GLP-2 are synergistically help the organism respond to nutrient availability but their main target differ. 
Teduglutide slows down proximal motility of the GIT, drives crypt cell proliferation by facilitation of the receptors and thus it increases the development of enterocytes, regenerates the intestinal musosa and helps enlarge the mucosal surface by rising villus height that shrink in absence of enteral feeding, on a whole it drive restoration of integrity of the gut wall [6].

Clinical results of use of teduglutide are fairly good: $20-24 \%$ of patients on exclusive Total Parenteral Nutrition (TPN) can get rid of it and more than half of the patients can decrease the dependence on daily TPN [7]. The success depends mostly on the remaining size of ileum and colon. Who has no one centimetre of these gut segments has no chance to improve gut adaptation with teduglutide therapy because the cells producing this hormone and its receptors are missing after the resection.

Nevertheless, beside the benefits due to the facilitation of the enterocytes accidental developments of polyps and increased tumorigenesis has been detected. Therefore a careful monitoring is required in SBS patients being on teduglutide therapy.

\section{Selection of Parenteral Amino Acids}

Amino Acids (AAs) are essential component of parenteral nutrition admixtures. As amino acids are crystalline and provision of combination of minimally 12-14 amino acids is needed to ensure building bricks for endogenous protein synthesis, industrially manufactured amino acid mixtures are used to make parenteral nutrition admixtures aseptically in the hospital pharmacy laboratory. After the 1980s, most of European hospital pharmacies had an aseptic „mixing unite” preparing individual parenteral nutrition mixtures. These laboratories, due to the introduction of industrial parenteral mixtures ( 2 and 3 chamber bags or „convenience systems”) have been closed, mostly based on uneconomical operation. Today, hospital based individual parenteral admixtures are present in the USA in a proportion of ca. $65-70 \%$. In Europe, majority of Total Parenteral Nutrition (TPN) is provided in form of industrially manufactured multichamber bags, which would be suitable for ca. $82 \%$ of the patients. In case of the rest compromise is needed.

In the era of precision medicine, more attention is paid to the tailor-made therapeutic solutions. In this context personalized nutrition admixtures would be more and more required, especially in the intensive care and, in the neonatology. This tendency has recently been started [8]. The composition of amino acids has an impact víz. the effectiveness of a given TPN is linked to the proportion of essential amino acids, rather than to the total AA content [9]. Recently a tendency to open mixing laboratories is detectable in Europe as well. Moreover, lately many publications support the fact that amino acids are underdosed in a remarkable mass of patients. Inadequate protein provision results in protein deficiency with extensive negative impact [10]. This bad practice partly numerous reasons, among others it can be deducted from the erroneous judgement that protein need is equal to amino acid need of a patient. However, due to the water production during the peptid bond formation $100 \mathrm{~g}$ amino acid intake results in $83 \mathrm{~g}$ protein only. In case of enteral nutrition, where the peptide- component is usually whey proteine, the loss is higher because the enterally administered protein must be decomposed to amino acids before endogenous protein synthesis covers the needed of new proteins.

\section{Selection of Fat Emulsions}

Fat emulsions are needed to ensure essential fatty acids, to increase caloric density of the nutrition admixtures and, since today fish oil is mandatory component of the lipid emulsion mixtures, to improve n3/ $\mathrm{n} 6$ ratio. Moreover, fish oil pure emulsion for parenteral use is available thus any TPN can be augmented with n3 Fatty Acids (FAs). The impact of $\mathrm{n} 3 \mathrm{FAs}$ in the prevention of inflammatory reaction mostly in the arachidonic cascade (competition of $n 6$ and $n 3$ fatty acids for the enzymes in their metabolic cascade) is well known. However, the importance of Eicosapentaenic Acid (EPA) and Docosapentaenic Acid (DHA) in the restoration of inflammatory process via resolvins and the partial prevention of release and action of inflammatory mediators by protectins and maresins are just recently recognized benefits. These discoveries are comparable benefits to those effected by the inhibition of proinflammatory reactions [11].

\section{Citrulline as Indicator}

In case of short bowel syndrome (status after removal of the majority of the gut) long term or life long parenteral nutrition (in form of home parenteral nutrition) is needed for majority of patients due to lack of absorbtive surface for food/nutrients. As certain adaptation of the remaining piece of gut exists: after a while many patients will be able to take up certain amounts of nutrients enterally thus partially can be feed again enterally or orally. The time frame and the extent of this adaptation is uncertain because the state of the gut could not be measured and clinicians regularly make challenge to see the patients reaction to enteral feeding. Some 10 years ago it has been discovered that the non-essential amino acid citrulline can be used as indicator of gut function [12]. Citrulline is produced almost exclusively by the duodenum and the upper intestinal (jejunal) enterocytes from glutamine and arginine. It has been documented that serum-citrulline (se-C) levels are in close correlation with the full amount of enterocytes thus with the function of the jejunum which is the main field of nutrient absorption. Randomized Clinical Studies (RCTs) and meta-analyses of RCTs demonstrated that the need of PN is inversely correlate with the se-C and so this can display the odds for the successfull enteral nutrition $[13,14]$. This discovery has multiple benefits because by this technique EN-challenges, that are uncomfortable for the patients, become causeless and the the $\mathrm{PN}$-dependence of the patient decreases moreover the cost of EN is much lower than that of PN.

\section{Enteral Nutrition}

Enteral nutrition (tube feeding) was and still is the first choice administration route in the medical nutrition if patients are not able to drink/eat per mouth proper amounts of macro- and micronutrients. As even recent studies confirm that the measured and /or calculated amounts of nutrients are not taken up orally/enterally by significant number of partients, supplemental nutrition is required. 


\section{Supplemental PN to EN}

In the last two-three decades enteral nutrition bacame declared as optimal route of feeding in patients not being able to feed orally. The old routin was that patients get either EN or, if EN was not enough or got impossible, PN. This line-up has fundamentally changed during the past 4-6 years [15]. It has been demonstrated, that most of the patients in the intensive care units don't get the calculated (needed) amounts of nutrients. Reasons are restricted absorption, high residual volumen, poor motility, etc. In case patients can be insufficiently nourished enterally, Supplemental Parenteral Nutrition (SPN) is necessary. The only way of supplying further protein and sources of energy in such cases is administration of supplemental intravenous delivery of nutrients. By this way one can administer higher amounts of nutrients and pharmaconutrients as well [16]. As most patients on enteral nutrition have no central venous access, this type of medical nutrition can also be administered as peripheral parenteral nutrition. SPN is a safe and cost-effective way of supplying the missing energy and amino acids for patients having nutritional deficits after enteral nutrition [17]. Randomized clinical trials demonstrated that short term catabolism can be stopped in high proportions of severly malnourished (intensive care) patients by this hybrid way of nutrition with additional benefits in decrease of nosocomial infections as well [18]. SPN can and should be given to non-intensive care patients as well, if nutritional data indicate it. This type of additional nutrition usually indicated transitionally only.

\section{Bioavailability of AA}

In patients with undernutrition protein catabolism dominates and during their nutrition support they get artificial protein sources (EN-formulas) to ensure successful endogenous protein synthesis preferably by oral or enteral nutrition. Unfortunately, in many hospitals patients do not receive sufficient protein supplementation $[19,20]$. Among the many reasons misconception could play a role, too. In the past clinicians calculated with $100 \%$ bioavailability. The measured or calculated need of the patient had to be cover with identical proteincontent of nutrition solutions. In this case declared protein-content of the nutrition solution was applied as basis for the calculation of necessary dose. Recent studies draw attention to the wastage in nutrients during the enteral nutrition. In 2002, van der Schoor and his team published the study to demonstrate the splanchnic first-pass effect affecting the bioavailability of the ingested protein but in the past 20 years clinicians and dieticians forgot about this loss [21]. Liebau and co-workers recently demonstrated the discrepancies between amounts of amino acids enterally administered and appeared in patients systemic circulation [22]. This loss may reach $15-20 \%$ as well. In this light the hyperalimentation concept of the sixties-seventies of the last century was not a big failure in the field of enteral and oral medical nutrition. Moreover, this is the time to rethink calculation technics of the daily routine in medical enteral nutrition and to use more frequently SPN.

\section{Citrulline Fortification}

Several efforts have been made to fortify enteral nutrition formulas with non-essential amino acids and conditionally essential amino acids in order to improve anabolic effect of feeding tube meals. One important example is arginin-enrichment. This type of pharmaconutrition is especially favorable in acceleration of wound healing [23,24]. Unfortunately, arginine has a high immediate metabolism in the liver (first pass effect) therefore the dose has been elevated in time. The successfull arginine-fortified formulas have in certain aspects some negative results because in high doses many patients presented adverse or toxic reactions. The change of arginine to citrulline could avoid these adverse effects, because it is direct precursor of arginine and the switch to citrulline resulted in much higher arginine blood levels than arginine administartion. Citrulline has exceptionally high bioavailability, too. Its urinary loss is minimal in comparison to arginine. Moreover, citrulline administration improved systemic amino acid availability, in genereal [25]. Further, clinical studies demonstrated its positive effects in sarcopenia and cardiovascular diseases, the latter is under investigations yet [26]. According tot he recent publications it seems citrullin will enter into the composition of EN formulas in the near future.

\section{Discussion}

Clinical nutrition is regarded as stepchild in the medical therapy, however its benefits are not to be queried. Its development is part of the global progress of medicine.

Identification of undernutrition is essential part of the patient hospital admisson. Here we displayed the novel globally accepted tool called GLIM criteria to diagnose undernutrition. This is an important advancement in the frame of international research activity because earlier the undernutrition determined on various assessment base could not ensure the comparability of the study results.

Parenteral nutrition is the most effective mode of artificial nutrition. Its unique benefit is that it can be used in patients with gastrointestinal failure. Typical example for dependence on parenteral nutrition is the increasing number of Short Bowel Syndrome (SBS). These patients often use PN life long (home parenteral nutrition), but some of them (who has more than 1,5-2m gut) are able to adapt their intestine to higher absorption rate within several months or years. This adaptation can be accelarated by the hormonal GLP-2 agonist teduglutide. Integration of this medicine into the treatment of SMS patients gives the chance of weaning off $\mathrm{PN}$ in a certain proportion of patients. Whether the induction of gut enteocytes successfull is, measurement of serum level of circulating citrulline became a good indicator. Both innovation basically influence home parenteral nutrition care and the quality of life of patients.

Macronutrients plays a pivotal role in medical nutrition therapy. Determination how much macronutrients (energy and protein sources) are needed to restore the patients' anabolism after the disease-induced catabolism needs sophisticated measurements and calculations. Recent recognitions helped clinicians to refine computation of optimal amino acid supplementation. Some of the macronutrients also have pharmacological activity as well therefore this type of nutrition is called pharmaconurtition. In case of lipid emulsions new research results opened new vistas in fighting against inflammation. By this way optimal combination of various 
fat emulsions may improve effectiveness of medical nutrition intervention. But there are news in the field of the first choice medical nutritional mode, the EN as well. Some misbeliefs were elucidated recently. The accurate control of patients on EN revealed that there are several reasons why patients don't get calculated amounts of EN on the wards. As adequate nutrition is prerequisit of proper healing, introduction of periperal parenteral nutrition for those who don't tolerate higher amounts ( $>80 \%$ of calory and protein need) of EN, is strongly recommended. Moreover the calculation of daily dose of enteral formulas should be changed due to the hidden loss of ingested protein source. Finally the impact of introduction of novel nutrients should be stressed. Recent appearance of precision medicine force the professionals of medicine and medical nutrition to reevaluate details of daily routine and the used tools, inclusive the medicines and enteral tube feeds. One can find the way to individualized nutrition as well, especially if nutrition support teams are working in the healthcare settings. Multidisciplinaty thinking bring the new ideas and the solutions.

\section{Summary}

Lifelong learning is imperativus for healthcare professionals as well. New materials and technics may improve medical diagnosis and medical interventions. Here we displayed some of the recent news in the sphere of medical nutrition. Use of teduglutide and citrulline improve quality of life of the short bowel patients. Recent news on amino acids and fat emulsions may provide better optimalization of parenteral nutrition therapy. Novelties in the field of enteral nutrition also contribute to better service within the healthcare system. This selection of news based on a subjective decision but for those not living in this medium may demonstrate the progress of a segment of clinical nutrition.

\section{References}

1. Jensen GL, Cederholm T, Correia MITD, Gonzalez MC, Fukushima R, et al. (2019) GLIM Criteria for the diagnosis of malnutrition: a consensus report from the global clinical nutrition community. Clin Nutr 39: 1-9. [crossref]

2. Fiorindi C, Luceri C, Dragoni G, Piemonti G, Scaringi S, et al. (2020) GLIM Criteria for malnutrition in surgical IBD patients: a pilot study. Nutrients 12: 2222. [crossref]

3. Allard JP, Keller H, Gramlich L, Jeejeebhoy KN, et al. (2020) GLIM Criteria has fair sensitivity and specificity for diagnosing malnutrition when using SGA as comparator. Clin Nutr 39: 2771. [crossref]

4. Keller H, de van der Schueren MAE, GLIM Consortium, Cederholm T, Barazzoni $\mathrm{R}$, et al. (2020) Global Liedership Initiative on Malnutrition (GLIM): Guidance on validation of the operational criteria for the diagnosis of protein-energy malnutrition in adults. JPEN 44: 992. [crossref]

5. Zhang X, Tang M, Zhang Q, Zhang K-P, Guo ZQ, et al. (2021) The GLIM criteria as an effective tool for nutrition assessment and survival prediction in older adult cancer patients. Clin Nutr 40: 1224. [crossref]

6. Kandjani OJ, Alizadeh AA, Moosavi-Movahedi AA, Kheradmand SS, et al. (2021) Expression, purification and molecular dynamics simulation of extracellular domain of glucagon-like peptide-2 receptor linked to teduglutide. Int J Biol Macromolecules 184: 812 .

7. Chen K, Joly F, Mu F, Kelkar SS, et al. (2021) Predictors and timing of response to teduglutide in patients with short bowel syndrome dependent on parenteral support. Clin Nutr 43: 420. [crossref]

8. Ginguay A, De Brandt J-P, Cynober, L (2016) Indication and contraindications for infusing specific amino acids (leucine, glutamine, arginine, citrulline and taurine) in critically illness. Curr Opin Clin Nutr Metab Care 19: 161-169. [crossref]
9. Iacone R, Scanzano C, Santarpia L, Cioffi I, Contaldo F, et al. (2020) Macronutrients in parenteral nutrition: amino acids. Nutrients 12: 772. [crossref]

10. Hsu C-C, Sun C-Y, Tsai C-Y, Chen C-Y, Wang SY, et al. (2021) Metabolism of proteins and amino acids in critically illness: from physiological alterations to relevant clinical practice. J Multidiscipl Healthcare 14: 11071117. [crossref]

11. Calder PC, Waitzberg DL, Klek S, Martindale RG (2020) Lipids in parenteral nutrition: biological aspects. JPEN 44: S21-27. [crossref]

12. Cynober L, Moinard C, De Bandt J-P (2010) The 2009 ESPEN Sir David Cuthbertson A new major signaling molecule or just another player in the pharmaconutrition game? Clin Nutr 29: 545-551. [crossref]

13. Jeppesen PB, Gabe SM, Seidner DL, Lee H-M, Olivier C (2020) Citrulline correlations in short bowel syndrome - intestinal failure by patient stratification: analysis of 24 weeks of teduglutide treatment from a randomized controlled study. Clin Nutr 39: 2479-2486. [crossref]

14. Proli F, Faragalli A, Talbotec C, Bucci A, et al. (2021) Variation of plasma citrulline as a predictive factor for weaning off long-term parenteral nutrition in children with neonatal short bowel syndrome. Clin Nutr 40: 4941-1947.

15. Oshima T, Pichard C (2015) Parenteral nutrition: never say never. Crit Care 19: S5. [crossref]

16. Singer P, Bendavid I, Mesilati-Stahy R, Green P, Rigler M, et al. (2021) Enteral and supplemental parenteral nutrition enriched with omega-3 polyunsaturated fatty acids in intensive care patients - a randomized controlled, double-blind clinical trial. Clin Nutr 40: 2544-2554. [crossref]

17. Pradelli L, Graf S, Pichard C, Berger MM (2018) Supplemental parenteral nutrition in intensive care patients: a cost saving strategy. Clin Nutr 37: 573-579.

18. Alsharif DJ, Alsharif FJ, Aljuraiban GS, Abulmeaty MMA (2020) Effect of supplemental parenteral nutrition versus enteral nutrition alone on clinical outcomes in critically ill adult patients: a systematic review and meta-analysis of randomized controlled trials. Nutrients 12: 2968. [crossref]

19. Osooli F, Abbas S, Farsaei S, Adibi P (2019) Identifying critically ill patients at risk of malnutrition and underfeeding: a prospective study at an academic hospital. $A d v$ Pharm Bull 9: 314. [crossref]

20. Rougier L, Preiser JC, Fadeur M, Verbrugge AM, et al. (2021) Nutrition during critical care: an audit on actual energy and protein intakes. JPEN 45: P951.

21. Van der Schoor SR, Reeds PJ, Stoll B, Henry JF, et al. (2002) The high metabolic cost of a functional gut. Gastroenterology 123: 1931-1940. [croosref]

22. Liebau F, Király E, Olsson D, Werneman J, et al. (2021) Uptake of dietary amino acids into anterial blood during continuous enteral feeding in critically ill patients and healthy subjects. Clin Nutr 40: 912-918.

23. Stechmiller JK, Childress B, Cowan L (2005) Arginine supplementation and wound healing. Nutr Clin Pract 20: 52-61.

24. Liu P, Shen W-Q, Chen H-L (2017) Efficacy of arginine-enriched enteral formulas for the healing of pressure ulcers: a systematic review. J Wound Care 26: 319-326. [crossref]

25. Bouillanne O, Mekchior J-C, Faure C, Paul M, Canouï-Poitrine F, et al. (2019) Impact of 3-week citrulline supplementation on postprandial protein metabolism in malnourished older patients: the Ciproage randomized controlled trial. Clin Nutr 38: 564-574. [crossref]

26. Papadia C, Osowska S, Cynober L, Forbes A (2018) Citrulline in health and disease. Review on human studies. Clin Nutr 37: 1823-1828. [crossref]

\section{Citation:}

Télessy IG (2021) Recent News in Medical Nutrition Therapy. Nutr Res Food Sci $J$ Volume 4(2): 1-4. 